\title{
Undergraduate students' perceptions and readiness: an evaluation of inter-professional education at Central Java, Indonesia
}

\begin{abstract}
This study aims to describe the perceptions of and readiness for IPE among nursing, medical and pharmacy students. The quantitative descriptive study was conducted in an IPE which held at the three faculties of a private university in Central Java, Indonesia. The IPE was delivered in two sessions with a seven-stage tutorial. The sample was taken using proportionate random sampling and engaged 83 of 425 students. A mean score analysis found that the mean value of students' perception was 88.04 (SD 6.72) for medical students, 84.66 (SD 10.26) for nursing and 81.14 (SD 10.41) for pharmacy students. Meanwhile, the pharmacy students have the highest readiness for the IPE followed by nursing and medical students with 73.45 (SD 7.85), 72.34 (SD 7.35) and 71.32 (SD7.36), respectively. Overall, the students have positive perceptions and readiness toward the program. It may be due to cognitive ability, culture effect, socialization, early clinical experience and IPE exposure in curricula.
\end{abstract}

Keyword: Inter-professional education; Perception; Readiness; Undergraduate healthcare student 